\title{
EVALUACIÓN DEL FUNCIONAMIENTO FAMILIAR CON EL FACES IV EN ADOLESCENTES CON PROBLEMÁTICA FAMILIAR
}

\author{
María I. Vegas Miguel \\ Universidad de Burgos \\ Raquel de la Fuente Anuncibay \\ Universidad de Burgos \\ raquelfa@ubu.es
}

Recepción Artículo: 13 marzo 2020

Admisión Evaluación: 17 marzo 2020

Informe Evaluador 1: 1 abril 2020

Informe Evaluador 2: 7 abril enero 2020

Aprobación Publicación: 20 abril 2020

Agradecimientos: Este trabajo ha sido financiado por la Consejería de Educación de la Junta de Castilla y León. Orden EDU/304/2019. (1licencia por estudios y otras actividades de interés para el sistema educativo, retribuida)

\section{RESUMEN}

Introducción: Las familias multiproblemáticas han recibido creciente atención por los poderes públicos, aunque hay una carencia de instrumentos específicos para evaluar su funcionamiento. En este estudio se propone el modelo Circunflejo de Olson (paquete FACES) como una herramienta de evaluación eficaz para este colectivo.

Método: se seleccionaron 49 adolescentes entre 14 y 18 años, con problemática familiar y se compararon sus resultados en funcionamiento familiar (FACES-IV), en comunicación y satisfacción familiar (mediante FCS y FSS), tiempo que comparten padres e hijos, número de veces que se come en familia, factores familiares estresantes y maltrato físico recibido, con un grupo general, compuesto por 857 adolescentes, seleccionados de 22 centros educativos de Burgos (España), mediante muestreo en dos etapas. En total: 46,5\% varones y 53,5\% mujeres.

Resultados: Ios adolescentes pertenecientes a familias problemáticas presentan menos cohesión familiar, menos flexibilidad, más desapego, más caos, menos comunicación y menos satisfacción familiar que la población general. Se caracterizan también por la falta de disponibilidad del padre, por comer menos veces en familia, haber sufrido más maltrato físico y ser las dificultades económicas y la conflictividad familiar, los principales estresores familiares.

Conclusión: el paquete FACES IV es un instrumento eficaz para la detección y evaluación de familias problemáticas y puede ser aplicado a adolescentes españoles de amplio espectro social y familiar.

Palabras clave: problemática familiar; evaluación; adolescencia; FACES IV; comunicación familiar 


\section{EVALUACIÓN DEL FUNCIONAMIENTO FAMILIAR CON EL FACES IV EN}

ADOLESCENTES CON PROBLEMÁTICA FAMILIAR

\section{ABSTRACT}

Family functioning assesment with faces iv in adolescents from problematic families. Introduction: Multiproblematic families have received increasing attention by public authorities, although there is a lack of specific instruments to assess its functioning. This paper proposes Olson's Circumflex model (FACES package) as an effective evaluation instrument for this group.

Method: The sample consisted of 49 Spanish teenagers from problematic families, aged between 14 and 18 , whose results in family functioning (FACES-IV), family communication (FCS), family satisfaction (FSS), time shared with parents, times a week they eat with family, family stressors and physical abuse suffered, were compared with a sample of 857 adolescents from 22 educative centres in Burgos (Spain), selected by a two-stage sampling. Altogether $46.5 \%$ men and $53.5 \%$ women.

Results: teenagers from problematic families show less family cohesion, less flexibility, less communication, less family satisfaction and their families are more disengaged and more chaotic than adolescents from general population. They are also characterized by the lack of availability of the father, by eating fewer times in family, by having suffered more physical abuse and by the economic difficulties and family conflicts being their main family stressors.

Conclusion: the FACES IV package is an effective instrument for detection and evaluation of problematic families and can be applied to Spanish adolescents from a broad social and family spectrum.

Keywords: problematic families; assessment; adolescents; FACES IV; family communication

\section{INTRODUCCIÓN}

Desde la segunda mitad del siglo XX ha habido una preocupación creciente por las familias que requerían apoyos formales de las instituciones y servicios sociales, las familias en riesgo de exclusión psicosocial o también denominadas familias multiproblemáticas (Linares \& Coletti, 1997), y en consecuencia, un afán por diseñar propuestas de intervención que tengan una fundamentación teórica sólida (Gómez, Mufioz, \& Haz, 2007). Sin embargo, el creciente interés no ha venido acompañado del desarrollo de herramientas de evaluación específicas para este colectivo (Jiménez, Lorence, Hidalgo, \& Menéndez, 2017).

Jiménez et al. definen familias en situación de riesgo psicosocial a "todas aquellas familias con un funcionamiento que compromete el bienestar de los menores, pero sin que la situación alcance un nivel de gravedad que justifique el desamparo y por tanto, la retirada de la custodia" (Jiménez et al., 2017, p. 2). Cuando el grado de gravedad de la disfunción parental es elevado, se han vulnerado los derechos del menor 0 existen evidencias de algún tipo de maltrato, se procede a la retirada de la guarda o tutela, de forma temporal o permanente. Generalmente estos menores, pasan a centros específicos, los denominados centros de protección o viven con otros jóvenes en pisos tutelados bajo la supervisión de un educador.

Pero para una evaluación profunda y comprehensiva de este tipo de familias, no podemos ceñirnos a dimensiones personales de cada miembro, sino ahondar también en dimensiones grupales y en las relaciones interpersonales que subyacen en el sistema familiar (Jiménez et al., 2017). La perspectiva ecológico-sistémica recoge este planteamiento y dentro de esta óptica, el modelo Circunflejo del Sistema marital y familiar (Olson, Russell, \& Sprenkle, 1979) ha desarrollado la Family Adapatability and Cohesion Evaluation Scale (FACES), uno de los cuestionarios más importantes a nivel internacional para evaluar el funcionamiento familiar, por su fundamentación teórica y su aplicabilidad en el ámbito clínico y en la investigación con familias (Hamilton \& Carr, 2016; Jiménez et al., 2017; Olson, 2000; Sanderson et al., 2009; White \& Klein, 2008).

El modelo circunflejo de Olson se basa en tres conceptos, considerados esenciales para el funcionamiento familiar:

a) la cohesión, definida como "Ios lazos emocionales que los miembros de una familia o pareja muestran entre si" (Olson, Waldvogel, \& Schlieff, 2019, p. 201).

b) la flexibilidad, definida como "la cantidad de cambio en el liderazgo, los roles y las reglas relacionales" (Olson et al., 2019, p. 202). 
c) La comunicación, considerada una dimensión facilitadora de las otras dos y descrita por Olson como "las habilidades de comunicación positiva utilizadas en el sistema familiar o conyugal' (OIson \& Gorall, 2003, p. 65).

Las principales hipótesis del modelo circunflejo de Olson son:

1. Las parejas y familias con niveles centrados ( 0 balanceados) en cohesión y flexibilidad, son más conducentes a un funcionamiento familiar saludable, mientras que niveles extremos (muy alto o muy bajo) en estas dos variables están asociados a funcionamiento familiar problemático (Olson \& Gorall, 2006).

2. Las familias y parejas equilibradas tienen más comunicación positiva que los sistemas no equilibrados (Olson et al., 2019).

Así pues, la comunicación, tal y como la describe Olson, tiene una relación lineal con el funcionamiento familiar o marital. Por tanto, cuanta más comunicación haya en la familia, mejor funcionará el sistema y viceversa. La comunicación familiar es evaluada a través de la Escala de Comunicación Familiar o Family Communication Scale (FCS) (Barnes \& Olson, 1985; Olson \& Barnes, 2010a).

La cohesión y la flexibilidad, sin embargo, tienen una relación curvilínea con el funcionamiento familiar, ya que valores extremos en alguna de las dos variables dan lugar a sistemas problemáticos o disfuncionales.

De ahí, que la última versión del modelo, el FACES IV, incluye dos variables balanceadas: cohesión balanceada y flexibilidad balanceada y cuatro dimensiones no balanceadas (Olson, 2011), que cubren la parte extrema (alta 0 baja) de ambas variables. Las variables no balanceadas relacionadas con la cohesión son Desapego (escasa cercanía emocional y excesiva independencia en los miembros de la familia) y Aglutinación (excesiva cercanía emocional y poca independencia). Las variables no balanceadas de la flexibilidad son Rigidez (muy poca capacidad de adaptarse a los cambios y excesivas normas, roles y reglas rígidos) y Caos (excesiva capacidad de cambio en los roles, reglas y liderazgo).

A estas escalas que cubren los tres constructos básicos del modelo circunflejo, Olson añadió más tarde la escala de Satisfacción Familiar o Family Satisfaction Scale (FSS) (Olson \& Barnes, 2010b; Olson et al., 2019), una forma de medir la satisfacción de las tres dimensiones principales del FACES IV.

La última versión del FACES IV ha sido validada en España para adultos (Martínez-Pampliega, Merino, Iriarte, \& Olson, 2017) y también se han comprobado las buenas propiedades psicométricas de una versión para adolescentes españoles.

Se han utilizado versiones anteriores del FACES para estudiar familias en riesgo de exclusión psicosocial (Jiménez et al., 2017) y se ha aplicado la última versión para el estudio de adolescentes italianos (Baiocco, Cacioppo, Laghi, \& Tafà, 2013; Everri, Mancini, \& Fruggeri, 2015, 2016). En España, se usó el FACES III para un trabajo sobre adolescentes en violencia-filioparental (Zuñeda, Llamazares, Marañón, \& Vázquez, 2016). Sin embargo, hasta el momento no se ha realizado ningún estudio empírico donde el FACES IV haya servido para detectar 0 evaluar el funcionamiento en adolescentes con problemática familiar, con riesgo de exclusión social 0 acogidos a un programa de protección. Y este precisamente es el objetivo del presente estudio, confirmar si las hipótesis de Olson se cumplen y si la escala de evaluación FACES IV aplicada a adolescentes con problemática familiar, sigue los niveles esperados en comunicación, satisfacción y en las escalas balanceadas y no balanceadas, comparados con adolescentes de la población general.

\section{METODOLOGÍA}

Se tomó una muestra de 906 adolescentes entre 14 y 18 años: 421 varones (46,5\%) y 485 mujeres (53,5\%). De ellos, 857 pertenecían a 22 centros educativos de la provincia de Burgos, y 49 a nueve centros de menores. El muestreo en los centros educativos se realizó en dos etapas, primero un muestreo estratificado en los centros y niveles educativos y después un muestreo por conglomerados, tomando como conglomerado cada clase seleccionada. Los 49 adolescentes de problemática familiar pertenecían a dos tipos diferentes de centros: por una parte, adolescentes que asisten a programas específicos para familias con riesgo de exclusión social y por otro lado, adolescentes acogidos en centros de protección. Participaron en la muestra todos aquellos adolescentes que de forma voluntaria y anónima aceptaron rellenar el cuestionario. En todos los casos, se elaboró un consen- 


\section{EVALUACIÓN DEL FUNCIONAMIENTO FAMILIAR CON EL FACES IV EN ADOLESCENTES CON PROBLEMÁTICA FAMILIAR}

timiento informado para el joven o sus padres y se actuó de acuerdo a la ley de Protección de Datos de carácter personal.

Se utilizó el FACES IV traducido y validado en España (Martínez-Pampliega et al., 2017), en una versión para adolescentes españoles. Las escalas de Flexibilidad balanceada, Cohesión balanceada y Desapego constaban cada una de los 7 ítems originales. Las escalas Rígidez y Caos tenían 5 ítems cada una y la escala de Aglutinación, 3 ítems. Para la comunicación familiar, se usó el cuestionario Family Communication Scale (FCS) y para la satisfacción familiar, la escala Family Satisfaction Scale(FSS). Ambos instrumentos validados en España (Sanz, Iraurgi, \& Martínez-Pampliega, 2002). Todas las escalas tenían respuesta tipo Likert, variando desde el 1 (no describe nada a mi familia / totalmente en desacuerdo) hasta el 5 (describe muy bien a mi familia /totalmente de acuerdo).

Para evaluar otras características del funcionamiento familiar, se realizaron otras preguntas de elaboración propia. Aquí se incluyeron cuestiones como "número de veces que comen a la semana en familia" y actividades que realizan conjuntamente padres e hijos, unas de tipo protección (por ej. "pedir ayuda cuando tienes un problema personal') y otras de tipo emocional (por ej. "expresar el cariño o el afecto que sientes"), así como situaciones familiares relevantes que habían creado tensión en la familia o en el adolescente. También se les preguntó si habían sufrido maltrato físico en la infancia.

Se calculó la estadística descriptiva en cada grupo: media, desviación típica y mediana. Para comparar las variables numéricas de ambos grupos y para prevenir la falta de normalidad en el grupo pequeño, se utilizaron las pruebas no paramétricas de Kruskall-Wallis para muestras independientes. Para comparar proporciones en las variables categóricas, se usó el estadístico Chi-cuadrado de Pearson y pruebas post-hoc Z con corrección de Bonferroni para las comparaciones múltiples. El análisis estadístico se llevó a cabo con el programa estadístico IBM SPSS Statistics 22.

\section{RESULTADOS}

\section{FACES IV}

Comparando el grupo de adolescentes con problemática familiar con el grupo general, se obtuvieron los siguientes resultados (ver tabla 1). Las diferencias significativas entre grupos aparecen reflejadas con un asterisCo. 
Tabla 1 Estadística descriptiva (media, desviación estándar y mediana) en las seis escalas del cuestionario FACES, para adolescentes con problemática familiar y de la población general

\begin{tabular}{llcc}
\hline & Media & $\begin{array}{c}\text { Adolescentes de } \\
\text { población } \\
\text { general }\end{array}$ & $\begin{array}{c}\text { Adolescentes con } \\
\text { problemática } \\
\text { familiar }\end{array}$ \\
\hline COHESIÓN* & Desviación estándar & 4,80 & 21,97 \\
& Mediana & 27,00 & 6,96 \\
\hline FLEXIBILIDAD** & Media & 24,94 & 23,50 \\
& Desviación estándar & 4,34 & 20,97 \\
& Mediana & 25,00 & 6,14 \\
\hline DESAPEGO* & Media & 16,68 & 22,08 \\
& Desviación estándar & 4,79 & 20,13 \\
& Mediana & 16,00 & 5,49 \\
\hline AGLUTINACIÓNN & Media & 8,05 & 20,00 \\
& Desviación estándar & 2,41 & 7,46 \\
& Mediana & 8,00 & 2,90 \\
\hline RÍGIDEZ & Media & 14,15 & 7,00 \\
& Desviación estándar & 3,67 & 14,67 \\
& Mediana & 14,00 & 4,85 \\
& Media & 10,91 & 15,00 \\
\hline CAOS* & Desviación estándar & 3,60 & 13,00 \\
& Mediana & 10,33 & 4,61 \\
& & & 12,50 \\
\hline
\end{tabular}

$(*)$ Escalas con diferencias significativas entre ambos grupos (p-valor $<0,05)$

Por tanto, los adolescentes con problemática familiar muestran menos cohesión familiar, menos flexibilidad, más caos y un sistema más desligado, es decir, con menos vínculos emocionales. Las diferencias en todas las variables son significativas y de gran intensidad (ver figura 1) Son comprensibles estos resultados, porque muchos de las disfunciones que presentan este tipo de familias son debidas a la falta afecto y al caos en jerarquía, liderazgo, normas y roles, así como a la falta de fronteras entre los subsistemas. Estos resultados coinciden con los estudios realizados sobre familias con problemática familiar. Rodríguez (2003) señala al caos organizativo como una de las peculiaridades de las familias multiproblemáticas, donde puede haber deficiencias en necesidades primarias, mientras pueden estar duplicados los electrodomésticos, por ejemplo. Gómez et al. (2007) proponen el abandono de las funciones parentales, el aislamiento y la desorganización como características centrales de este tipo de familias.

En las variables Aglutinado y Rígido, no se encontraron diferencias significativas entre ambos grupos. 
Figura 1: Diferencias en funcionamiento familiar, comunicación y satisfacción familiar entre adolescentes de familias problemáticas y del grupo general.

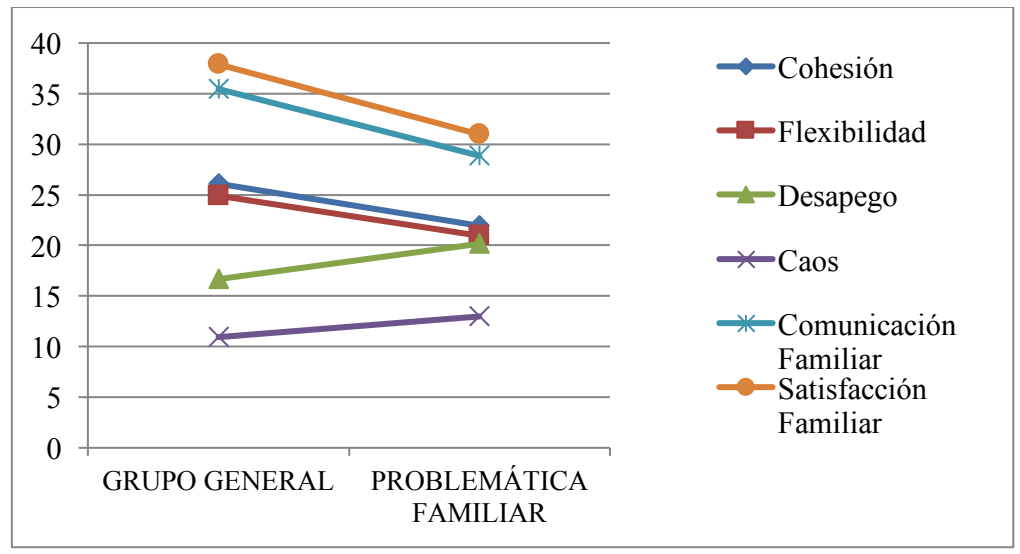

\section{Comunicación familiar y satisfacción familiar}

Comparando los resultados en comunicación y satisfacción familiar mediante los cuestionarios FCS y FSS, respectivamente, también se comprobó que había diferencias importantes y significativas (ver figura 1). La media en ambos grupos en comunicación familiar (población general =35,44; problemática familiar = 28,89) y en satisfacción familiar (población general = 37,93 ; problemática familiar = 30,97) indica que los adolescentes con problemática familiar muestran menor satisfacción familiar y están menos contentos con la comunicación que existe en la familia.

\section{Relación emocional y actividades realizadas con ambos progenitores}

Al comprobar el porcentaje de respuestas en los dos grupos a las preguntas sobre las actividades que realizan los adolescentes con ambos padres, se observaron las siguientes diferencias significativas: Ios adolescentes con problemática familiar realizaban menos actividades de tipo protección con el padre y menos actividades de contenido emocional con el padre, que la población general (ver figura 2). Así pues, parece que la ausencia del padre 0 su falta de disponibilidad es una de las características definitorias de este colectivo.

También los jóvenes con problemática familiar comen menos veces a la semana en familia (una media de 4,98 ) que los jóvenes del grupo general (media de 7,65). Es decir, estos últimos comen al menos una vez al día con su familia (ver figura 2), un tiempo distendido para compartir, conversar o ser escuchados, algo que no ocurre en los adolescentes con problemas familiares. 
Figura 2: Diferencias en actividades (de protección y tipo emocional) realizadas con el padre y en el número de comidas familiares, entre adolescentes de familias problemáticas y del grupo general.

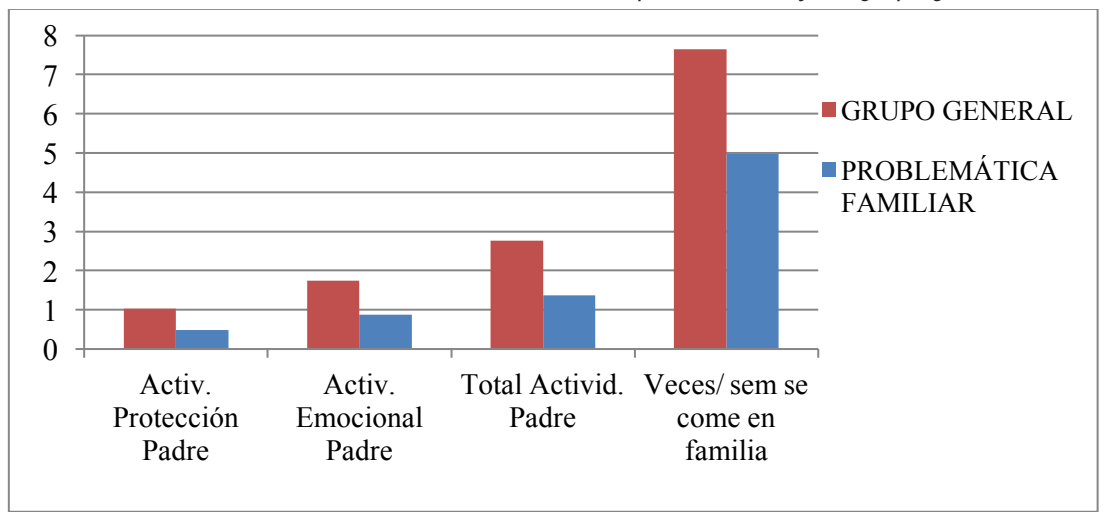

\section{Factores familiares estresantes}

En la tabla 2, se muestra el porcentaje de respuestas en cada grupo respecto a las circunstancias familiares que han podido ser generadores de estrés o tensión para ellos. Estas son las preguntas donde se obtuvieron diferencias significativas:

Tabla 2 Porcentaje de adolescentes en cada grupo que señalan determinadas situaciones familiares como generadoras de tensión para ellos.

\begin{tabular}{lccc}
\hline $\begin{array}{c}\text { Situaciones familiares } \\
\text { generadoras de } \\
\text { estrés /tensión }\end{array}$ & $\begin{array}{c}\text { Adolescentes } \\
\text { de población } \\
\text { general } \\
(\mathrm{n}=857)\end{array}$ & $\begin{array}{c}\text { Adolescentes de } \\
\text { familias en riesgo } \\
\text { psicosocial } \\
(\mathrm{n}=35)\end{array}$ & $\begin{array}{c}\text { Adolescentes en } \\
\text { centros de } \\
\text { protección } \\
(\mathrm{n}=14)\end{array}$ \\
\hline * Situación de paro & 8,7 & $31,4^{*}$ & 14,3 \\
* Dificultades económicas & 10,1 & $42,9^{*}$ & $35,7^{*}$ \\
* Discusiones padres/ hijos \\
frecuentes
\end{tabular}

(*) Diferencias significativas con el grupo de la población general.

(**) Diferencias significativas con el grupo de la población general y con el grupo en riesgo psicosocial. 


\section{EVALUACIÓN DEL FUNCIONAMIENTO FAMILIAR CON EL FACES IV EN ADOLESCENTES CON PROBLEMÁTICA FAMILIAR}

Por tanto, la situación de paro es un problema que genera tensión en los adolescentes en riesgo psicosocial. Las dificultades económicas y el hecho de tener un familiar alcohólico provocan más tensión en los adolescentes con problemática familiar en general. Y los menores en centros de protección se caracterizan por no tener tiempo para compartir y ser escuchados en la familia y por una gran conflictividad familiar: un porcentaje altísimo de discusiones padres/hijos frecuentes, discusiones entre padres o por otros asuntos familiares. Además, consideran en mayor proporción que en la población general, que se les exige como hijos responsabilidades excesivas.

\section{MALTRATO FísICO}

Si en el grupo general, los adolescentes referían maltrato físico en un 6,1\% de los casos, uno de cada cuatro adolescentes con problemática familiar (24,5\%), afirmaban haber recibido maltrato físico; una diferencia abultada y estadísticamente significativa.

En la bibliografía citada por Linares y Coletti, se describe a familias en situación de pobreza y marginación social de la segunda mitad del siglo XX, precisamente por varias de las características obtenidas en este estudio: el alcoholismo, las drogodependencias, la delincuencia o los malos tratos (Linares \& Coletti, 1997; Rodríguez, 2003).

\section{CONCLUSIÓN}

Se aplicó la escala de evaluación familiar FACES IV a adolescentes españoles que vivían en un contexto de problemática familiar. En esto grupo se incluyeron jóvenes que pertenecían a familias en riesgo de exclusión social y otros menores que estaban acogidos en centros de protección. Y se confirmaron casi todas las hipótesis planteadas por Olson y colaboradores (Olson, 2011; Olson et al., 2019), a saber: que estas familias con disfunción familiar presentaban menor nivel de comunicación familiar que familias de la población general. También las escalas balanceadas del FACES IV, Cohesión y Flexibilidad, obtenían menor puntuación, mientras algunas de las escalas no balanceadas, en concreto Desapego y Caos, tenían niveles más altos que las familias de la población normal. Solo las escalas de Aglutinamiento y Rigidez no mostraban diferencias con la población general.

También se observó que en las familias problemáticas, las veces que se come en familia, así como el tiempo empleado por los progenitores con el adolescente, en concreto por el padre, era menor que en los adolescentes pertenecientes al grupo general. El maltrato físico, las dificultades económicas y los conflictos familiares (padres/hijos, entre padres o con el resto de la familia) eran características diferenciadoras de los adolescentes que viven en familias problemáticas.

Como era previsible, Ios adolescentes de familias problemáticas decían estar menos satisfechos con sus familias.

Estos datos confirman la disfuncionalidad adjudicada a este tipo de familias y corroboran la evaluación eficaz del funcionamiento familiar a través del cuestionario FACES IV, ya que las percepciones manifestadas por los adolescentes en este cuestionario coincidían con las valoraciones realizadas por los servicios sociales.

Así pues, puede concluirse que el paquete FACES IV - compuesto por el cuestionario FACES IV, la escala de comunicación familiar (FCS) y la escala de Satisfacción Familiar (FSS) - es un instrumento eficaz para detectar familias que presentan un funcionamiento familiar problemático o disfuncional y puede ser aplicado a adolescentes españoles de amplio espectro social y familiar.

Entre las limitaciones de este trabajo es el escaso número de adolescentes pertenecientes a familias problemáticas en comparación con la población general, debido a la dificultad para identificar a estos menores, y muy especialmente, para convencer a éstos y a sus familias para participar en el estudio y rellenar el cuestionario.

En futuros estudios también será importante seguir investigando las escalas de Aglutinamiento y Rigidez en adolescentes, ya que éstas no cumplen las hipótesis planteadas por Olson sobre las escalas no balanceadas. 


\section{REFERENCIAS BIBLIOGRÁFICAS}

Baiocco, R., Cacioppo, M., Laghi, F. y Tafà, M. (2013). Factorial and Construct Validity of FACES IV Among Italian Adolescents. Journal of Child and Family Studies, 22(7), 962-970.

Barnes, H. L. y Olson, D. H. (1985). Parent-adolescent communication and the circumplex model. Child Development, 56, 438-447.

Everri, M., Mancini, T. y Fruggeri, L. (2015). Family Functioning, Parental Monitoring and Adolescent Familiar Responsibility in Middle and Late Adolescence. Journal of Child and Family Studies, 24(10), 3058-3066.

Everri, M., Mancini, T. y Fruggeri, L. (2016). The Role of Rigidity in Adaptive and Maladaptive Families Assessed by FACES IV: The Points of View of Adolescents. Journal of Child and Family Studies, 25(10), 2987-2997.

Gómez, E., Mufioz, M. M., \& Haz, A. M. (2007). Familias Muitiproblematicas y en Riesgo Social: Caracteristicas e Intervencion. Psykhe, 16(2), 43-54.

Hamilton, E. y Carr, A. (2016). Systematic Review of Self-Report Family Assessment Measures. Family Process, 55(1), $16-30$.

Jiménez, L., Lorence, B., Hidalgo, V. y Menéndez, S. (2017). Análisis factorial de las escalas FACES (Family Adaptability and Cohesion Evaluation Scales) con familias en situación de riesgo psicosocial. Universitas Psychologica, 16(2).

Linares, J. L. y Coletti, M. (1997). La intervención sistémica en los servicios sociales ante la familia multiproblem: La experiencia de Ciutat Vella. Barcelona: Paidós. Terapia Familiar.

Martínez-Pampliega, A., Merino, L., Iriarte, L. y Olson, D. H. (2017). Propiedades psicométricas de la versión española de la escala de evaluación de la adaptabilidad y la cohesión familiar. Psicothema, 29(3), 414-420.

Olson, D. H., Russell, C. S. y Sprenkle, D. H. (1979). Circumplex Model of Marital and Family Systems Il: Empirial Studies and Clinical Intervention. (J. Vincent, Ed.), Advances in Family Intervention. Assessment and Theory. Greenwich, Conn. JAI Press.

Olson, D. H. (2000). Circumplex Model of Marital and Family Systems. Journal of Family Therapy, 22, 144-167.

Olson, D. H. y Gorall, D. M. (2003). Circumplex Model of Marital and Family Systems. In F. Walsh (Ed.), Normal Family Processes. (3rd. Edition) (pp. 514-547). New york: Guilford.

OIson, D. H. y Gorall, D. M. (2006). FACES IV \& the circumplex model. Life Innovations, Inc.

Olson, D. H. y Barnes, H. L. (2010a). Family Communication Scale. Minneapolis. MN: PREPARE/ENRICH, LLC.

OIson, D. H. y Barnes, H. L. (2010b). Family Satistaction Scale. Minneapolis. MN: PREPARE/ENRICH, LLC.

Olson, D. H. (2011). FACES IV and the Circumplex Model: Validation Study. Journal of Marital and Family Therapy, 37(1), 64-80.

Olson, D. H., Waldvogel, L. y Schlieff, M. (2019). Circumplex Model of Marital and Family Systems: An Update. Journal of Family Theory and Review, 11(2), 199-211.

Rodríguez, M. (2003). La familia multiproblemática y el modelo sistémico. Portularia: Revista de Trabajo Social, (3), 89-115.

Sanderson, J., Kosutic, I., Garcia, M., Melendez, T., Donoghue, J., Perumbilly, S., Perumbilly, S., Franze, C. y Anderson, S. (2009). The measurement of outcome variables in couple and family therapy research. American Journal of Family Therapy, 37(3), 239-257.

Sanz, M., Iraurgi, I., \& Martínez-Pampliega, A. (2002). Evaluación del funcionamiento familiar en toxicomanías: Adaptación española y características de adecuación métrica del FAP-FACES IV. In I. Iraurgi \& F. González (Eds.), Instrumentos de Evaluación en drogodependencias. (pp. 403-434). Madrid: Aula Médica.

White, J., \& Klein, D. (2008). Family Theories (3rd. ed.). California: Sage Publications.

Zuñeda, A., Llamazares, A., Marañón, D., \& Vázquez, G. (2016). Características individuales y familiares de los adolescentes en violencia filio-parental: la agresividad física, la cohesión familiar y el conflicto interparental como variables explicativas. Revista de Psicopatología y Psicología Clínica, 21(1), 21-33. 
